\title{
Impact of POD modes energy redistribution on flow reconstruction for unsteady flows of impulsively started airfoils and wings
}

\author{
G. Pascarella ${ }^{\mathrm{a}}$, M. Fossati ${ }^{\mathrm{a}}$ and G. Barrenechea ${ }^{\mathrm{b}}$ \\ aAerospace Centre of Excellence, University of Strathclyde, 75 Montrose St., Glasgow G1 \\ 1XJ, UK \\ ${ }^{\mathrm{b}}$ Dep. of Mathematics and Statistics, University of Strathclyde, 26 Richmond St., Glasgow \\ G1 1XJ, UK
}

ARTICLE HISTORY

Compiled February 17, 2019

\begin{abstract}
Obtaining accurate CFD solutions of unsteady flows during the design process of an aircraft can be a highly-demanding task in terms of computational and time resources. A common practice is the recourse to Reduced Basis Methods (RBM), which manage to reduce the number of degrees of freedom to be solved yet allow preserving high accuracy, as opposed for example to low-fidelity methods like vortexlattice or panel methods. RBM based on Proper Orthogonal Decomposition have been extensively studied and adopted but limitations are observed when trying to solve unsteady problems, where the temporal sequence of snapshots and the evolving nonlinear dynamics of the flow field need to be addressed carefully while building the reduced model. The present work investigates the problem of the accuracy in reconstructing nonlinear unsteady fluid flows by means of RBM methods for a specific class of impulsively started lifting bodies. The classical snapshot POD approach and a recent variant named Spectral POD will be comparatively studied to assess their capacity to reconstruct unsteady flow fields typical of aerospace applications. The periodic motion past a cylinder will be considered first as a benchmark test while the impulsive start of a $2 \mathrm{D}$ three element airfoil and a 3D wing in high-lift configurations will be considered as use cases.
\end{abstract}

\section{KEYWORDS}

Reduced Order Modeling; Proper Orthogonal Decomposition; Spectral Proper

Orthogonal Decomposition; Impulsive Start; Flow Reconstruction

\section{Introduction}

The investigation of unsteady aerodynamics is a critical element in the design of an aircraft and it can be a really time-consuming task, especially when many different aircraft configurations or operating conditions have to be assessed. In the attempt to reduce the computational requirements of these analyses while preserving strong consistency with the physics, the so-called Reduced Basis Methods (RBM) have been developed. The literature is dense with contributions that cover different types of flows and different aspects of the reduction process. Notable introductions to the topic can be found in the work of Rowley and Dawson (2017), Taira et al. (2017), Lassila et al. (2014) and Dowell et al. (1999). RBM allow obtaining accurate aerodynamic

CONTACT G. Pascarella Author. Email: gaetano.pascarella@strath.ac.uk 
solutions in a fast manner by extracting relevant knowledge from a set of available solutions (called snapshots or observables) in the form of basis functions of a vector space (also referred to as modes) and using it to recast the original problem in a more compact form, where the number of degrees of freedom (DoF) is substantially reduced. The whole process has two phases: an off-line and on-line steps. The modes identification step is regarded as an off-line phase since it is performed once and for all, using the information coming from either simulations or experiments. The on-line phase is instead the step where the physical solution is reconstructed by combining together the information brought by the modes. Both steps are crucial since defining a good RBM implies identifying the correct low-dimensional space and describing properly its dynamics and/or its evolution with changing parameters over this space.

For the specific case of unsteady flows, the off-line phase can be extremely delicate since it needs to define a proper sub-space where the nonlinear time dynamics is completely embedded. The loss of important dynamical information at this stage might cause misleading results and/or instabilities during the on-line phase, which need to be fixed in some ways, usually introducing additional terms in the model. This might happen when relying for example on Proper Orthogonal Decomposition (POD) (Weller, Lombardi and Iollo 2009; Cazemier 1998) to identify the RBM modes. POD was introduced for the analysis of turbulent flows by Lumley (Lumley 1967) and it has been widely used in literature for both steady and unsteady problems (Stabile and Rozza 2018; Ripepi et al. 2018; Stabile et al. 2017; Ripepi and Goertz 2015; Zhan, Habashi and Fossati 2015; Iuliano and Quagliarella 2013; Carlberg and Farhat 2008; Lieu, Farhat and Lesoinne 2005; Bui-Thanh 2003; Legresley and Alonso 2000), since it allows to describe most of the information of the initial data set with the lowest number of modes possible (Holmes et al. 2012). It identifies a basis, which is the closest to the set of snapshots in terms of an average projection error based on an energy norm. The POD optimality has to be intended as the ability to describe most of the energy content of the initial data set with the lowest number of modes possible. Nevertheless, POD considers no correlation among the snapshots of the system at the feature extraction level. Each snapshot is treated as a statistical realization of the system under consideration and stochastically independent from all the others. This might be a strong limit when unsteady problems are considered and the evolution over time is strongly nonlinear. The statistical approach of POD might results in the loss of important dynamical information (Rowley 2005), which are usually not the most likely realization of the system (the most energetic ones).

A lot of effort has been put in the literature to overcome this recognized limit. The Dynamic Mode Decomposition (DMD) (Schmid 2010) was introduced as a method intended to extract dynamic information also and not only spatial structures from the snapshots. Once the dynamic modes are extracted from the data, the system dynamics can be reconstructed using an analytic formula where time appears explicitly, without the need to rely on the governing equation of the initial system or other techniques to recover time dynamics. The DMD procedure to approximate the time dynamics relies only on the super-position of dynamic modes oscillating in time with a particular frequency and grow/decay rate. This might become a source of inaccuracy when flows with no characteristic pattern in frequency are considered. It is worth to notice that DMD is equivalent to Discrete Fourier Transform (DFT) when applied to a data-set with zero-mean (Chen, Tu and Rowley 2012). Attempts to overcome the limits of POD and DMD for complex, non-periodic unsteady flows, led to the introduction of the so-called Spectral Proper Orthogonal Decomposition (SPOD) (Sieber, Paschereit and Oberleithner 2016), that introduces a filter at the basis extraction level, which 
explicitly allows taking into account the snapshots temporal correlation. The filter can be applied over a time window which varies continuously from an interval of zero-size to the whole observed time-interval. This allows SPOD to be a bridge between POD, i.e. filter of zero-size, and DMD/Discrete Fourier Transform (DFT) when the filter is applied over the whole time-interval. In the last case, the modes time dynamics is in fact dictated by sines and cosines as it would be for DFT. As such, SPOD aims at identifying a bridge between the ability to obtain an optimal reduction of the system and the need to preserve the nonlinear dynamic information coming from data (Noack 2016). The main effect of the SPOD filter applied at the basis extraction stage is to change the energy levels associated to the modes which define the reduced basis. The energy level of each mode is defined on the basis of the norm used for the basis extraction.

The aim of the present work is to investigate how the energy redistribution induced by the SPOD extraction technique over the reduced basis can influence the reconstruction of particular unsteady aeronautical flows, namely the dynamics of the starting vortices detaching from airfoil and wings with complex geometries consequently to an impulsive start. The square cylinder is also considered to evaluate the different performances of POD and SPOD when a von Karman vortex street is established. The paper is structured as follows: in Sections 2 and 3 the offline and on-line phases of the RBM techniques used, namely POD and SPOD, are briefly described, in Section 4 the techniques are applied to the chosen test-cases and results are presented, finally in Section 5 a discussion and conclusions are reported.

\section{Proper Orthogonal Decomposition and Spectral POD}

In this section, POD and SPOD are briefly introduced with particular attention to the crucial differences on the basis of which the two methods will behave in reconstructing the dynamics of unsteady flows. In the following, only data coming from CFD simulation will be considered as snapshots. A reduced basis method allows to express the unsteady solution $\mathbf{u}(\mathbf{x}, t)$ of the original system in terms of an combination of modes

$$
\mathbf{u}(\mathbf{x}, t) \approx \hat{\mathbf{u}}(\mathbf{x}, t)=\sum_{i=1}^{N_{m}} a_{i}(t) \phi_{i}(\mathbf{x})
$$

with $N_{m}$, number of modes, smaller than the number of initial snapshots $N_{s}$, used to build the RBM, and much smaller than the number of initial DoF coming from the CFD simulation. The $\phi_{i}$ are the modes which define the low-dimensional basis, while the $a_{i}$ are the coefficients required to reconstruct the physical solution starting from the modes. They can be provided by intrusive or non-intrusive techniques.

POD allows to extract an optimal basis from a given set of snapshots in terms of a specific norm, defined over the $n$-dimensional space spanned by the snapshots themselves. For a CFD simulation $n$ is the number of grid points defined by the mesh times the number of unknowns chosen to build the reduced basis. The norm is taken as the measure of the global kinetic energy of each flow snapshot

$$
\|\mathbf{u}\|=\sqrt{\sum_{i=1}^{n} u_{i}^{2}}
$$


where $\mathbf{u}$ is the vector of unknowns, which is a unique column vector with all the velocity components at each grid point, $\mathbf{u}=[u, v, w]^{T}$. Therefore, the POD optimality condition, which allows to extract the closest basis to the initial dataset, is defined

$$
\max _{\Phi \in \mathbb{R}^{n}}\langle\mathbf{U}, \Phi\rangle \quad \text { with } \quad\|\Phi\|=1
$$

where $\langle\cdot, \cdot\rangle$ represents the average over time and $\mathbf{U}$ is the matrix of the collected snapshots $\left\{\mathbf{u}_{1}, \mathbf{u}_{2}, \ldots, \mathbf{u}_{N_{s}}\right\}$. In order to extract POD modes $\boldsymbol{\Phi}$, the method of snapshots introduced by Sirovich (Sirovich 1987) has been largely used in literature, especially for fluid dynamics problems, since it is based on solving an eigenvalue problem that is much smaller than the initial number of degree of freedom. According to Sirovich, since POD modes can be expressed as a linear combination of the initial snapshots

$$
\phi_{i}(\mathbf{x})=\sum_{i=1}^{N_{s}} a_{i} \mathbf{u}_{\mathbf{i}}
$$

the procedure used to extract POD modes is equivalent to solving the following eigenvalue problem

$$
\mathbf{R a}_{i}=\lambda_{i} \mathbf{a}_{i}
$$

where $\mathbf{R}$ is the POD temporal correlation matrix, defined as $\mathbf{R}=\mathbf{U}^{*} \mathbf{U}, \lambda_{i}$ are the POD eigenvalues, which are a measure of the energy associated to the corresponding mode, and $\mathbf{a}_{i}$ are the POD eigenvectors, which bring information about the time dynamics. At this point it can be noticed how the dimension of the eigenvalue problem to solve is $N_{s}$, number of snapshots used to build the POD basis, much smaller than $n$, DoF. The spatial modes can be recovered then as

$$
\phi_{i}=\frac{1}{\sqrt{\lambda_{i}}} \mathbf{U} \mathbf{a}_{i}
$$

The idea of Spectral POD is to introduce a filter applied to the correlation matrix $\mathbf{R}$, which leads to the following filtered matrix

$$
R_{f_{i, j}}=\sum_{k=-N_{f}}^{N_{f}} g_{k} R_{i+k, j+k}
$$

where $N f$ is the size of the SPOD filter, $g_{k}$ are weights. Equation (7) shows that the SPOD filter acts along the diagonal of the temporal correlation matrix, which is equivalent to weight elements characterized by the same $\Delta t$ in time, i.e. the function $R\left(t_{1}, t_{2}\right)$ is evaluated for different $t_{1}$ and $t_{2}$, keeping $t_{1}-t_{2}=\Delta t$ constant (Sieber, Paschereit and Oberleithner 2017). This procedure leads to an energy redistribution within the modes with respect to POD, which will be shown to be useful to recover a better dynamics. The filter $N_{f}$, which is the SPOD tuning parameter, allows to make a balance between the most likely states of the system, i.e. the most energetic ones provided by the POD, promoting the dimensionality reduction, and the temporal correlation between snapshots, which is important to preserve the dynamics of less energetic structures. The weights $g_{k}$ can be defined in different ways, but the most 
reasonable choice seems to be either a box filter with $g_{k}=\frac{1}{1+N_{f}}=$ const. or a Gaussian filter with certain variance (Sieber, Paschereit and Oberleithner 2016). Once the $\mathbf{R}_{f}$ matrix is computed, the procedure to extract spatial and temporal modes proceeds in the same way as POD. It is worth to notice that, when the filter in Equation (7) acts over all the correlation matrix, $\mathbf{R}_{\mathbf{f}}$ becomes a Toeplitz matrix and the SPOD is equivalent to a DFT (Gray 2006; Wise 1955). Therefore in the following, when the SPOD is performed using $N_{f}$ equal to the size of the matrix $\mathbf{R}$, it will be referred to as either DFT or SPOD.

\section{Reconstruction}

To obtain the coefficients for reconstruction in Equation (1) at each desired time instant an intrusive or non-intrusive technique can be used. The intrusive technique is a projection technique where the system governing equations are first projected into the extracted low-dimensional basis and then integrated using the classical methods for solving ODEs or PDEs. The non-intrusive technique consists instead of a datafitting approach based on the available snapshots. Most of the literature about RBM for unsteady problems uses intrusive techniques while non-intrusive techniques are more often considered for parametric studies. In the present work, a non-intrusive procedure is used to obtain the coefficients, to avoid some of the known issues of projection based approaches when dealing with compressible viscous flows (Rowley, Colonius and Murray 2004; Weller, Lombardi and Iollo 2009; Barone et al. 2009). Radial basis functions are considered

$$
a_{j}(t) \approx \sum_{i=1}^{N_{s}} w_{i} f\left(\left\|t-t_{i}\right\|\right)
$$

where the $t_{i}$ are the time instants corresponding to the components of the POD eigenvectors $\mathbf{a}_{i}$ in Equation (5).

\section{Results}

Three test cases are considered to evaluate the ability in reconstruction of the POD and the recent SPOD introduced in Section 2. Two different categories of flows are considered, namely a flow with periodic oscillations and an unsteady impulsive start flow without any characteristic frequency. The improved ability of the SPOD is highlighted in reconstructing the second category of flows over the all time interval investigated, once the energetic content is fixed, as opposed to the case of a periodic motion, where POD and SPOD are only slightly different. The energetic content of the reduced basis as a function of the number of modes $N_{m}$ is computed as

$$
E_{c}=\frac{\sum_{i=1}^{N_{m}} E_{i}}{\sum_{i=1}^{N_{s}} E_{i}} \times 100=\frac{\sum_{i=1}^{N_{m}} \lambda_{i}}{\sum_{i=1}^{N_{s}} \lambda_{i}} \times 100
$$




\begin{tabular}{c|cccccc}
\hline$N_{f}$ & $\begin{array}{l}N_{m} / N_{s} \\
E_{n}=99.9 \%\end{array}$ & $\epsilon_{u}(\%)$ & $\epsilon_{v}(\%)$ & $\begin{array}{l}N_{m} / N_{s} \\
E_{n}=95 \%\end{array}$ & $\epsilon_{u}(\%)$ & $\epsilon_{v}(\%)$ \\
\hline \hline 0 (POD) & $22 / 30$ & 1.5 & 4.0 & $6 / 30$ & 3.4 & 8.4 \\
\hline 10 & $28 / 30$ & 1.4 & 3.8 & $10 / 30$ & 4.3 & 9.4 \\
\hline 20 & $29 / 30$ & - & - & $12 / 30$ & 2.7 & 7.5 \\
\hline 30 (DFT) & $29 / 30$ & - & - & $13 / 30$ & 2.0 & 5.7 \\
\hline
\end{tabular}

Table 1. Error in reconstruction for the square cylinder, computed according to Equation (10) for $u$ and $v$ component of velocity, for two different energetic content and varying the SPOD filter size.

For each test-case, the snapshots are obtained using the SU2 CFD solver and the global error in reconstruction, defined as

$$
\epsilon=\frac{\left\|\mathbf{u}_{C F D}-\mathbf{u}_{R B M}\right\|}{\left\|\mathbf{u}_{C F D}\right\|}
$$

is reported for a specific time instant, where the $\mathbf{u}_{R B M}$ is the reduced basis reconstruction obtained with either POD or SPOD, fixing the energetic content in Equation (9), whereas $\mathbf{u}_{C F D}$ is the exact CFD solution. Moreover, qualitative results in terms of reconstruction are reported as well, comparing the contour lines of the RBM and CFD solution. All the reconstructed time instant are computed out of the training points, in order to test how the RBM performs in reconstructing new solutions, i.e. not used to build the reduced basis.

Square Cylinder. The periodic flow past a square cylinder is considered. The solution is initialized using the far field conditions and the sampling of the snapshots to build the reduced basis is carried out after the initial transient, once the vortex shedding has established. The domain discretization is performed through a completely structured mesh with 190244 number of elements and 191040 number of points. A dual time-stepping method is used for the unsteady simulation with $\Delta t=1.5 \times 10^{-3} \mathrm{~s}$ and the Mach number, Reynolds number and angle of attack are respectively $M=0.1$, $R e=22000$ and $\alpha=0 \mathrm{deg}$. At the fixed Reynolds number, the vortex street is turbulent, therefore the simulation is run as fully turbulent using SST turbulence model. To build the RB, a sampling $\Delta t$ equal to four times the $\Delta t_{C F D}$ is fixed and a number of snapshots $N_{s}=30$ is sampled over the limit cycle, equispaced in time. The time required to compute one single time step of the CFD simulation is approximately 0.5 $\mathrm{h}$ on one core, considering for the dual-time stepping $10^{-6}$ as the residuals tolerance and a maximum of 1000 inner iterations. For both POD and SPOD, the time required is approximately $0.3 \mathrm{~s}$ for off-line phase and $0.12 \mathrm{~s}$ to obtain a single reconstructed snapshot for the on-line phase. Table 1 reports the error for the two components of velocity as defined in Equation (10) and the number of modes required for two fixed energetic contents, namely $95 \%$ and $99.9 \%$. Figures 1 and 2 report instead contours of the exact and reconstructed solution.

30P30N airfoil. The impulsive start of the three component 30P30N airfoil is now investigated (Rumsey et al. 1998). The solution is initialized using the far field conditions and the sampling of the snapshots to build the reduced basis is performed from the first step of the simulation. The mesh used for the domain discretization is a viscous unstructured mesh with 559652 number of elements and 327733 number of points. A dual time-stepping method is used for the unsteady simulation with $\Delta t=10^{-3} \mathrm{~s}$ 


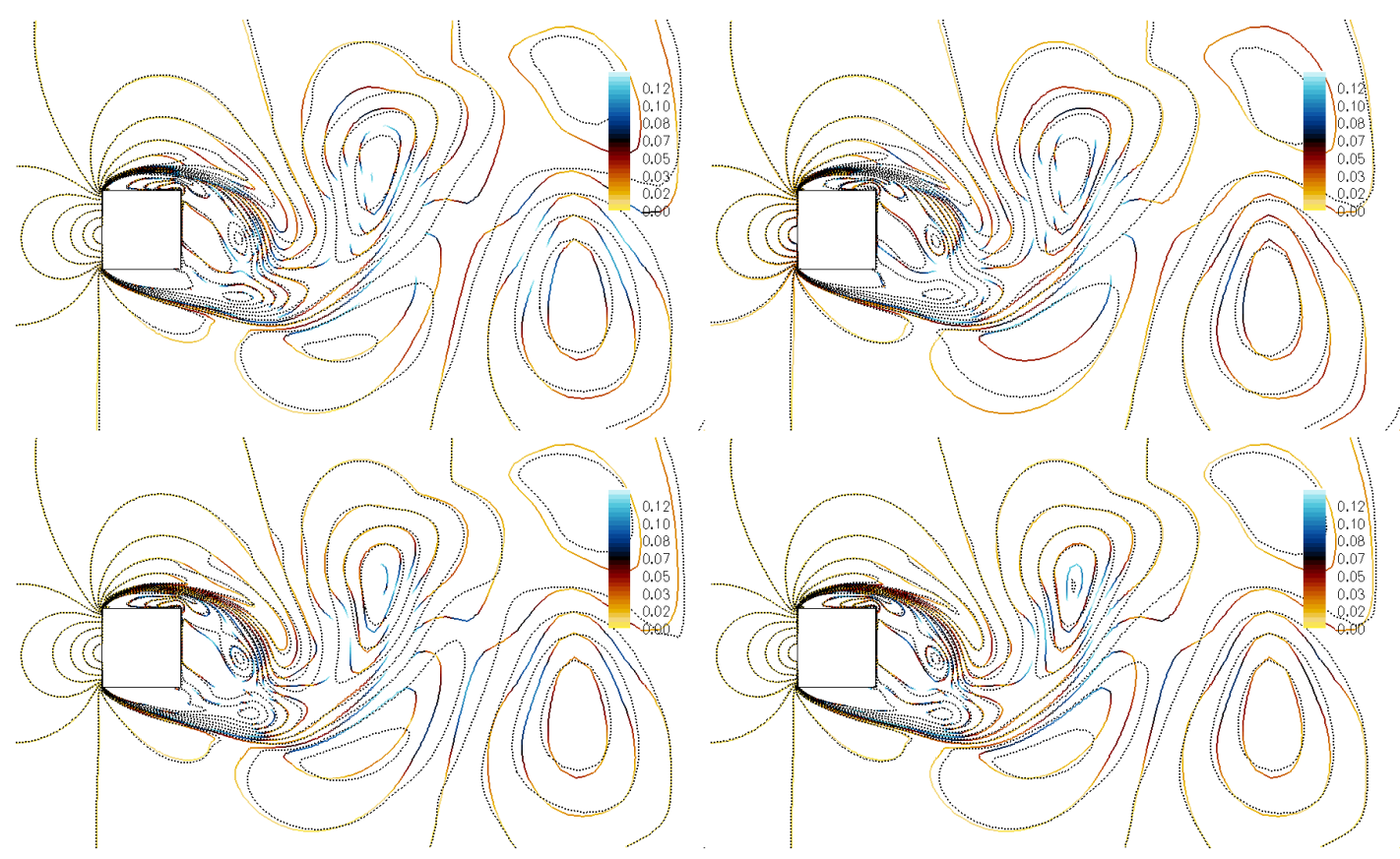

Figure 1. Comparison between velocity magnitude contours from CFD and RBM reconstruction with different values of the SPOD filter, using $E_{c}=95 \%$ at time $t=3.5 \mathrm{~s}$ from the impulsive start (left to right, top to bottom: $\left.N_{f}=0,10,20,30\right)$. Solid line: CFD coloured with the value of the error $\epsilon$; Dashed line: RBM reconstruction.

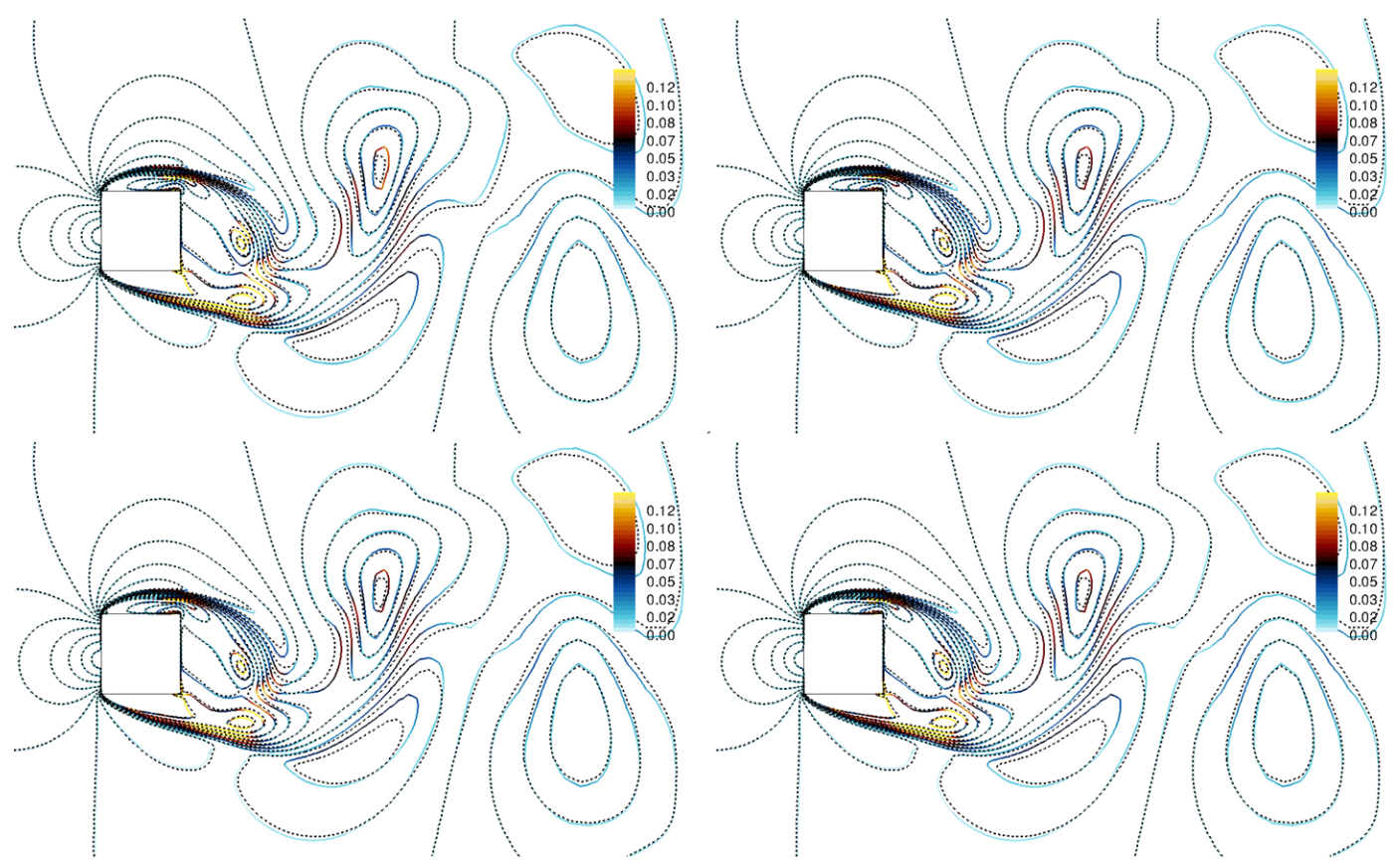

Figure 2. Comparison between velocity magnitude contours from CFD and RBM reconstruction with different values of the SPOD filter, using $E_{c}=99.9 \%$ at time $t=3.5 \mathrm{~s}$ from the impulsive start (left to right, top to bottom: $\left.N_{f}=0,10,20,30\right)$. Solid line: CFD coloured with the value of the error $\epsilon$; Dashed line: RBM reconstruction. 


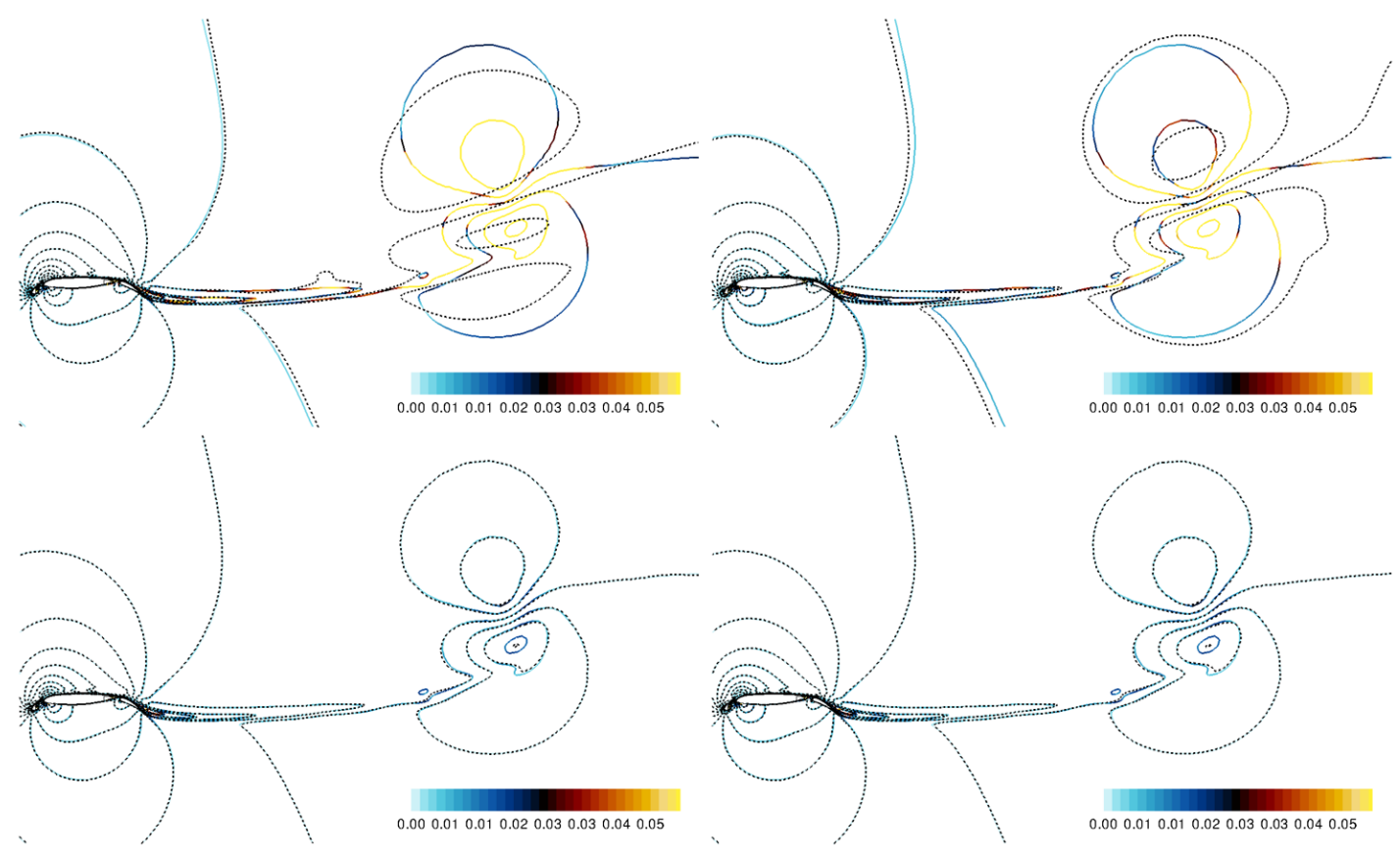

Figure 3. Comparison between velocity magnitude contours from CFD and RBM reconstruction with different values of the SPOD filter, using $E_{c}=95 \%$ at time $t=6.5 \times 10^{-2} \mathrm{~s}$ from the impulsive start (left to right, top to bottom: $\left.N_{f}=0,10,30,40\right)$. Solid line: CFD coloured with the value of the error $\epsilon$; Dashed line: RBM reconstruction.

\begin{tabular}{c|cccccc}
\hline$N_{f}$ & $\begin{array}{l}N_{m} / N_{s} \\
E_{n}=99.9 \%\end{array}$ & $\epsilon_{u}(\%)$ & $\epsilon_{v}(\%)$ & $\begin{array}{l}N_{m} / N_{s} \\
E_{n}=95 \%\end{array}$ & $\epsilon_{u}(\%)$ & $\epsilon_{v}(\%)$ \\
\hline \hline $0(\mathrm{POD})$ & $20 / 40$ & 0.4 & 0.98 & $7 / 40$ & 2.8 & 4.9 \\
\hline 10 & $32 / 40$ & 0.3 & 0.72 & $14 / 40$ & 1.7 & 3.2 \\
\hline 20 & $35 / 40$ & 0.2 & 0.53 & $18 / 40$ & 0.8 & 1.9 \\
\hline 30 & $38 / 40$ & - & - & $22 / 40$ & 0.5 & 1.2 \\
\hline $40(\mathrm{DFT})$ & $40 / 40$ & - & - & $25 / 40$ & 0.5 & 1.2 \\
\hline
\end{tabular}

Table 2. Error in reconstruction for the 30P30N, computed according to Equation (10) for $u$ and $v$ component of velocity, for two different energetic content and varying the SPOD filter size.

and the Mach number, Reynolds number and angle of attack are respectively $M=0.2$, $R e=9 \times 10^{6}$ and $\alpha=19 \mathrm{deg}$. The turbulence is modeled using SST turbulence model. The RB is built using a sampling $\Delta t$ equal to three times the $\Delta t_{C F D}$ and a number of snapshots $N_{s}=40$ equi-spaced in time. The time required to compute a single time step for the CFD simulation is approximately $1.25 \mathrm{~h}$ on one core, considering for the dual-time stepping $10^{-6}$ as the residuals tolerance and a maximum of 1000 inner iterations. For both POD and SPOD, the time required is approximately $0.7 \mathrm{~s}$ for the off-line phase and $0.32 \mathrm{~s}$ to obtain a single reconstructed snapshot for the on-line phase. Table 2 reports the error for the two components of velocity as defined in Equation (10) and the number of modes required for two fixed energetic contents, namely $95 \%$ and $99.9 \%$. Figures 3 and 4 report instead contours of the exact and reconstructed solution.

High-Lift wing-body configuration. The impulsive start of a high-lift wing body configuration is finally considered (Rumsey et al. 2011). Only half of the geometry is 


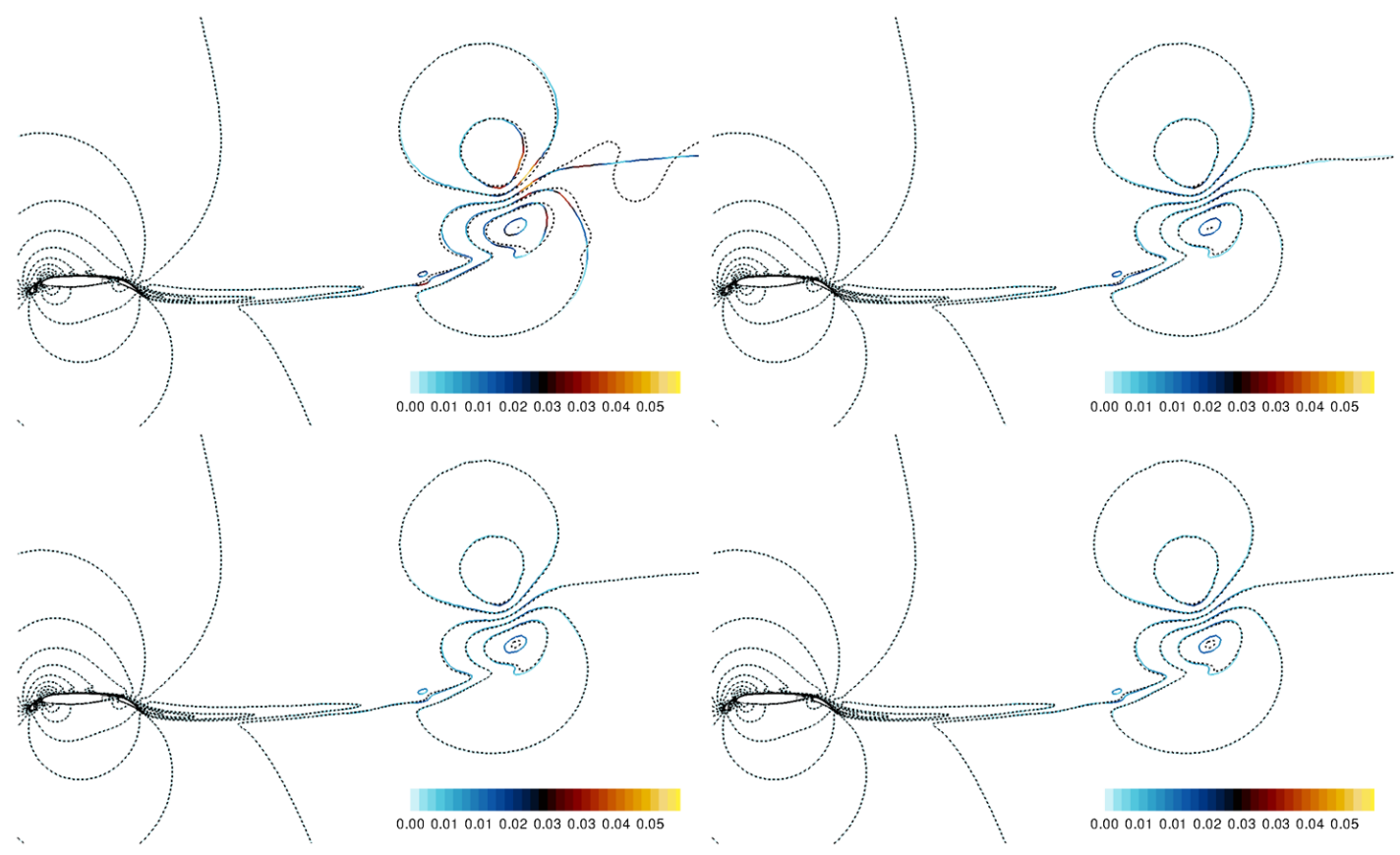

Figure 4. Comparison between velocity magnitude contours from CFD and RBM reconstruction with different values of the SPOD filter, using $E_{c}=99.9 \%$ at time $t=6.5 \times 10^{-2} \mathrm{~s}$ from the impulsive start (left to right, top to bottom: $\left.N_{f}=0,10,30,40\right)$. Solid line: CFD coloured with the value of the error $\epsilon$; Dashed line: $\mathrm{RBM}$ reconstruction.

retained and a symmetry boundary condition is imposed on the plane of symmetry. The mesh used is a viscous unstructured mesh with 21492137 number of elements and 3652657 number of points. Analogously to the previous test-case, the solution is initialized using the far field conditions and the sampling of the snapshots to build the reduced basis is performed from the first step of the simulation. A dual time-stepping method is used for the unsteady simulation with $\Delta t=10^{-3} \mathrm{~s}$ and the Mach number, Reynolds number and angle of attack are respectively $M=0.2, R e=4.3 \times 10^{6}$ and $\alpha=13 \mathrm{deg}$. The turbulence is modeled using SST turbulence model. To build the RB, a sampling $\Delta t$ equal to six times the $\Delta t_{C F D}$ is fixed and a number of snapshots $N_{s}=18$ is sampled, equi-spaced in time. The time required to compute a single time step for the CFD simulation is approximately $80 \mathrm{~h}$ on one core, considering for the dual-time stepping $10^{-6}$ as the residuals tolerance and a maximum of 1000 inner iterations. For both POD and SPOD, the time required is approximately $12 \mathrm{~s}$ for the off-line phase and $8.2 \mathrm{~s}$ to obtain a single reconstructed snapshot for the on-line phase. Table 3 reports the error for the three components of velocity as defined in Equation (10) and the number of modes required for a fixed energetic content of $99.9 \%$. Figures 5 and 6 report instead contours of the exact and reconstructed solution on two different slices in span wise direction.

\section{Discussion and final remarks}

The use of the two RBM introduced in Section 2 to reconstruct new approximate solutions reduces the computational time of several order of magnitude with respect to the full order model. The energy redistribution due to the filter applied to the POD correlation matrix entails a greater number of modes to reach a fixed energetic 


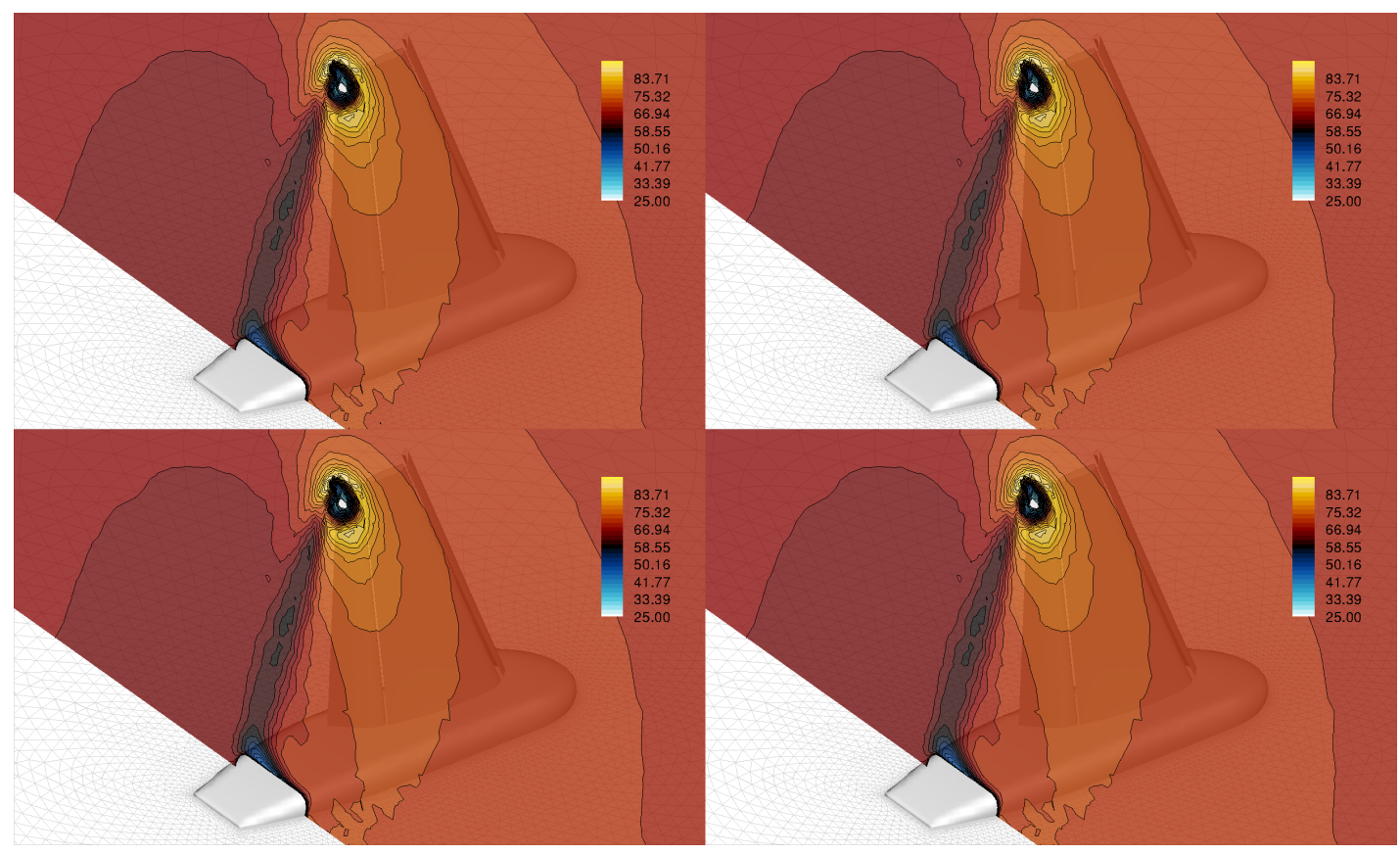

Figure 5. Comparison between velocity magnitude contours from CFD and RBM reconstruction with different values of the SPOD filter, using $E_{c}=99.9 \%$ at time $t=8.1 \times 10^{-2} \mathrm{~s}$ from the impulsive start (left to right, top to bottom: $\left.N_{f}=0,6,12,18\right)$. Filled contours: CFD velocity magnitude; Solid black lines: RBM reconstruction.

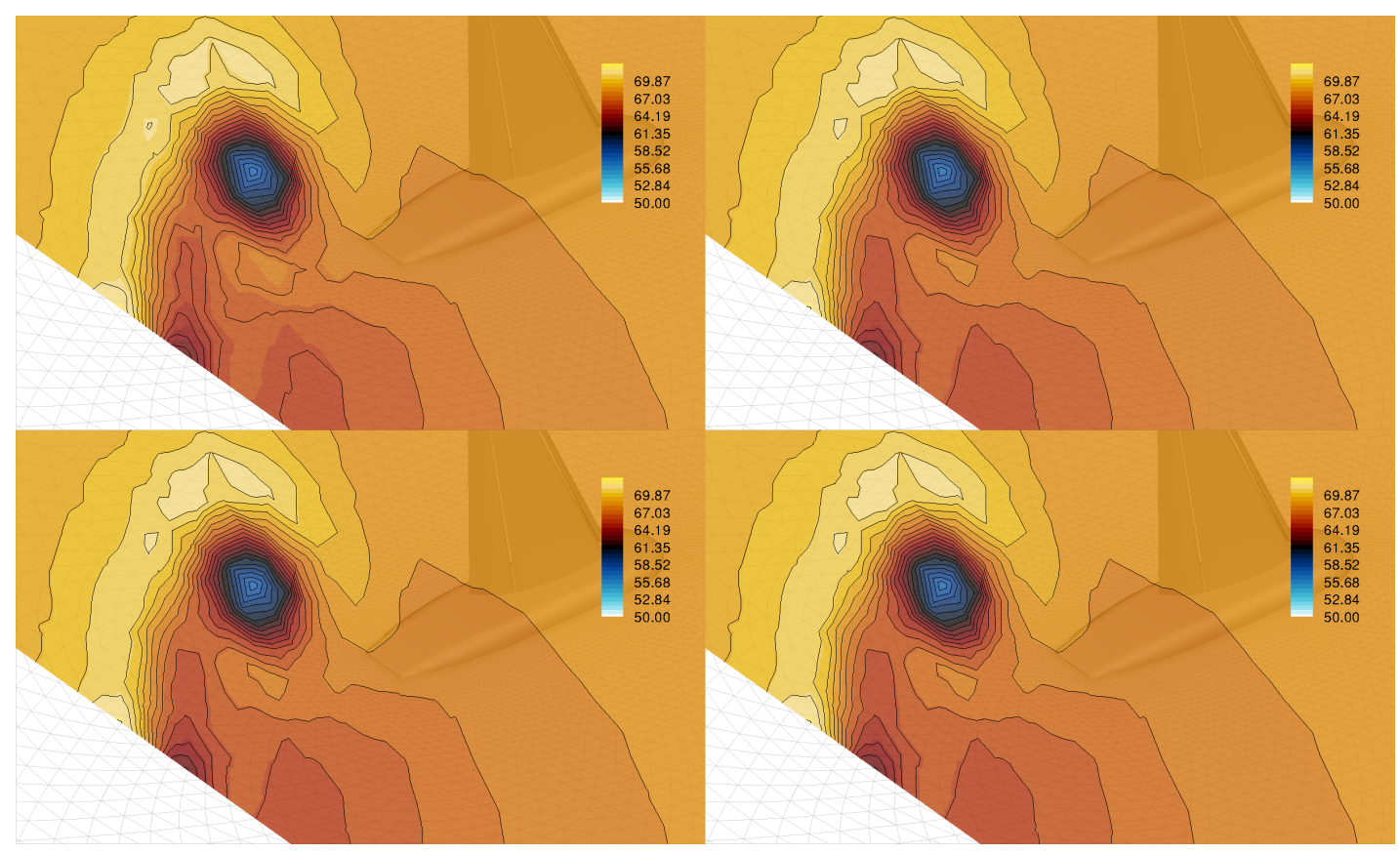

Figure 6. Comparison between velocity magnitude contours from CFD and RBM reconstruction with different values of the SPOD filter, using $E_{c}=99.9 \%$ at time $t=8.1 \times 10^{-2} \mathrm{~s}$ from the impulsive start (left to right, top to bottom: $\left.N_{f}=0,6,12,18\right)$. Filled contours: CFD velocity magnitude; Solid black lines: RBM reconstruction. 


\begin{tabular}{c|cccc}
\hline$N_{f}$ & $\begin{array}{l}N_{m} / N_{s} \\
E_{n}=99.9 \%\end{array}$ & $\epsilon_{u}(\%)$ & $\epsilon_{v}(\%)$ & $\epsilon_{w}(\%)$ \\
\hline \hline 0 (POD) & $14 / 18$ & 1.20 & 1.35 & 3.65 \\
\hline 6 & $17 / 18$ & 1.22 & - & - \\
\hline 12 & $18 / 18$ & - & - & - \\
\hline 18 (DFT) & $18 / 18$ & - & - & - \\
\hline
\end{tabular}

Table 3. Error in reconstruction for the 3D wing-body configuration, computed according to Equation (10) for $u, v$ and $w$ component of velocity, with fixed energetic content and varying the SPOD filter size.

content for each test-case, as it can be noticed from Tables 1, 2 and 3. Nevertheless, the computational cost of the two techniques is almost the same, both for the offline and on-line steps, since the main dimensionality reduction is performed in terms of considering at most $N_{s}$ modes, instead of the $n$ initial DoF coming from the CFD simulation. There is no significant effect on the global error as the filter is varied for the three test-cases but differences can be noticed locally, looking at the contour fields. For the periodic case, vortex shedding past a square cylinder, an energetic content of $99.9 \%$ shows no difference between POD and SPOD (Figure 2). However, as the energetic content is lowered to $95 \%$ (Figure 1) a fine difference can be noticed between the two techniques in resolving the coherent structures more downstream in the von Karman vortex street.

For the case of the impulsive start of the 30P30N airfoil, the same behaviour can be highlighted for both the energetic content (Figure 3 and 4). The POD doesn't perform as well as SPOD in reproducing the dynamic of the starting vortex as it propagates downstream and dissipates. The difference between the two techniques is much more remarkable when an energetic content of $95 \%$ is fixed (Figure 3), where it can be noticed how the performances in resolving the starting vortex dynamics improve significantly as the filter increases. Finally, for the 3D wing-body configuration, the Figures 5 and 6, corresponding to an energetic content of $99.9 \%$, highlight again how the POD fails in reconstructing the whole dynamics of the starting vortex. As a matter of fact, Figure 6 shows how the reconstruction improves as the filter increases in the sense that more details of the flow field are recovered. This behaviour, common over the three test-cases, can be easily explained considering the optimization procedure on which POD basis extraction is performed. Since the POD modes represent a set which maximize in average the energy of projection of the set of initial snapshots, coherent structures which are less energetic might not be recovered properly. This can be clearly seen comparing the two slices reported in Figures 5 and 6 for the 3D test-case. Closer to the wing, where the coherent structures have an higher energetic content, POD is able to recover the flow field with the same level of detail as SPOD. On the slice far away from the wing, instead, the coherent structures are less energetic and SPOD performs better.

Downstream of these observations the following conclusions can be drawn:

- The energy-based POD mode truncation has limitations in recovering the whole unsteady dynamics of less energetic structures;

- It is relevant to take into account the temporal dependency of snapshots at the modes extraction level (SPOD filter applied to the temporal correlation matrix);

- The energy-based SPOD mode truncation allows a better reconstruction of less energetic flow structure dynamics. 


\section{Acknowledgement(s)}

The authors wish to acknowledge the useful discussion with Mr. Tezzele and Mr. Demo from Prof. Rozza's group at SISSA. CFD results were obtained thanks to the EPSRC funded ARCHIE-WeSt High Performance Computer (www.archie-west.ac.uk). EPSRC grant no. EP/K000586/1.

\section{References}

Barone M. F., Kalashnikova I., Segalman D. J. and Thornquist H. K. 2009. "Stable Galerkin reduced order models for linearized compressible flow". Journal of Computational Physics 228(6), 1932-1946.

Bui-Thanh T., Damodaran M. and Willcox K. 2003. "Proper orthogonal decomposition extensions for parametric applications in compressible aerodynamics". In 21st AIAA Applied Aerodynamics Conference (p. 4213).

Carlberg K. and Farhat C. 2008. "A compact proper orthogonal decomposition basis for optimization-oriented reduced-order models". In 12th AIAA/ISSMO Multidisciplinary Analysis and Optimization Conference (p. 5964).

Cazemier W., Verstappen R. W. C. P. and Veldman A. E. P. 1998. "Proper orthogonal decomposition and low-dimensional models for driven cavity flows". Physics of fluids 10(7), 1685-1699.

Chen K. K., Tu J. H. and Rowley C. W. 2012. "Variants of dynamic mode decomposition: boundary condition, Koopman, and Fourier analyses". Journal of nonlinear science 22(6), 887-915.

Dowell E., Hall K., Thomas J., Florea R., Epureanu B. and Heeg J. 1999. "Reduced order models in unsteady aerodynamics". In 40 th Structures, Structural Dynamics, and Materials Conference and Exhibit (p. 1261).

Galletti B., Bruneau C. H., Zannetti L. and Iollo A. 2004. "Low-order modelling of laminar flow regimes past a confined square cylinder". Journal of Fluid Mechanics, 503, 161-170.

Gray R. M. 2006. "Toeplitz and circulant matrices: A review". Foundations and Trends in Communications and Information Theory 2(3), 155-239.

Holmes P., Lumley J. L., Berkooz G. and Rowley C. W. 2012. Turbulence, coherent structures, dynamical systems and symmetry. Cambridge university press.

Iuliano E. and Quagliarella D. 2013. "Proper orthogonal decomposition, surrogate modelling and evolutionary optimization in aerodynamic design". Computers Fluids 84, 327-350.

Lassila T., Manzoni A., Quarteroni A.and Rozza G. 2014. "Model order reduction in fluid dynamics: challenges and perspectives". In Reduced Order Methods for modeling and computational reduction (pp. 235-273). Springer, Cham.

Le Gresley P. and Alonso J. 2000. "Airfoil design optimization using reduced order models based on proper orthogonal decomposition". In Fluids 2000 conference and exhibit (p. 2545).

Liang Y. C., Lee H. P., Lim S. P., Lin W. Z., Lee K. H. and Wu C. G. 2002. "Proper orthogonal decomposition and its applicationsPart I: Theory". Journal of Sound and vibration 252(3), $527-544$.

Lieu T., Farhat C. and Lesoinne M. 2005. "POD-based aeroelastic analysis of a complete F-16 configuration: ROM adaptation and demonstration". In 46th AIAA/ASME/ASCE/AHS/ASC Structures, Structural Dynamics and Materials Conference (p. 2295).

Lumley J. L. 1967. "The structure of inhomogeneous turbulent flows." Atmospheric turbulence and radio wave propagation.

Noack B. R. 2016. "From snapshots to modal expansionsbridging low residuals and pure frequencies". Journal of Fluid Mechanics 802, 1-4.

Ripepi M., Verveld M. J., Karcher N. W., Franz T., Abu-Zurayk M., Grtz S. and Kier T. M. 
2018. "Reduced-order models for aerodynamic applications, loads and MDO". CEAS Aeronautical Journal 9(1), 171-193.

Ripepi M. and Goertz S. 2015. "Accelerating Unsteady CFD Simulations Using a Minimum Residual Based Nonlinear Reduced Order Modeling Approach". In Symposium on AeroStructures (pp. 237-255). Springer, Cham.

Rowley C. W. and Dawson S. T. 2017. "Model reduction for flow analysis and control". Annual Review of Fluid Mechanics 49, 387-417.

Rowley C. W. 2005. "Model reduction for fluids, using balanced proper orthogonal decomposition". International Journal of Bifurcation and Chaos 15(03), 997-1013.

Rowley C. W., Colonius T. and Murray R. M. 2004. "Model reduction for compressible flows using POD and Galerkin projection". Physica D: Nonlinear Phenomena 189(1-2), 115-129.

Rumsey C. L., Slotnick J. P., Long M., Stuever R. A. and Wayman T. R. 2011. "Summary of the first AIAA CFD high-lift prediction workshop". Journal of Aircraft 48(6), 2068-2079.

Rumsey C. L., Gatski T. B., Ying S. X. and Bertelrud A. 1998. "Prediction of high-lift flows using turbulent closure models". AIAA journal 36(5), 765-774.

Schmid P. J. 2010. "Dynamic mode decomposition of numerical and experimental data". Journal of fluid mechanics $656,5-28$.

Sieber M., Paschereit C. O. and Oberleithner K. 2017. "On the nature of spectral proper orthogonal decomposition and related modal decompositions". arXiv preprint arXiv:1712.08054.

Sieber M., Paschereit C. O. and Oberleithner K. 2016. "Spectral proper orthogonal decomposition". Journal of Fluid Mechanics 792, 798-828.

Sirovich L. 1987. "Method of snapshots". Quarterly of applied mathematical 45(3), 561-571.

Stabile G.and Rozza G. 2018. "Finite volume POD-Galerkin stabilised reduced order methods for the parametrised incompressible NavierStokes equations". Computers Fluids.

Stabile G., Hijazi S., Mola A., Lorenzi S. and Rozza G. 2017. "POD-Galerkin reduced order methods for CFD using Finite Volume Discretisation: vortex shedding around a circular cylinder". Communications in Applied and Industrial Mathematics 8(1), 210-236.

Taira K., Brunton S. L., Dawson S. T., Rowley C. W., Colonius T., McKeon B. J., Schmid O. T., Gordeyev S., Theofilis V. and Ukeiley L. S. 2017. "Modal analysis of fluid flows: An overview." Aiaa Journal 4013-4041.

Weller J., Lombardi E. and Iollo A. 2009. "Robust model identification of actuated vortex wakes". Physica D: nonlinear phenomena 238(4), 416-427.

Wise J. 1955. "The autocorrelation function and the spectral density function". Biometrika $42(1 / 2), 151-159$.

Zhan Z., Habashi W. G. and Fossati M. 2015. "Local reduced-order modeling and iterative sampling for parametric analyses of aero-icing problems". AIAA Journal 53(8), 2174-2185. 